\title{
The Therapeutic Effect of Melatonin on GC by Inducing Cell Apoptosis and Autophagy Induced by Endoplasmic Reticulum Stress
}

This article was published in the following Dove Press journal: OncoTargets and Therapy

\section{Yanshan Zheng \\ Jiawei Tu \\ Xinxin Wang \\ Yue Yu \\ Jiajia Li \\ Yin Jin* \\ Jiansheng $\mathrm{Wu}^{*}$}

Department of Gastroenterology, The First Affiliated Hospital of Wenzhou Medical University, Wenzhou 325000, People's Republic of China

*These authors contributed equally to this work
Correspondence: Jiansheng $\mathrm{Wu}$; Yin Jin Department of Gastroenterology, The First Affiliated Hospital of Wenzhou Medical University, Wenzhou 325000, People's Republic of China

Tel +8657755559191

EmailwzwujsIII@I63.com; wzjinyin@I63.com
Background: Gastric cancer (GC) is the main malignancy affecting a large population worldwide. Lack of effective enough treatment is one of the leading factors contributing to the high mortality rate. Melatonin, a naturally occurring compound, has been proven to exert cytotoxic and antiproliferative effects on human gastric cancers. Nevertheless, the mechanisms of anti-gastric cancer of melatonin remain elucidated. It is believed that endoplasmic reticulum (ER) stress and its resultant unfolded protein response (UPR) are connected to the survival, progression, and chemoresistance of various tumor cells via multiple cellular procedures, such as autophagy. In this study, the effects of melatonin on human gastric cancer cell lines AGS and SGC-7901 was assessed to reveal the interaction between melatonin, endoplasmic reticulum stress, and autophagy in gastric cancer.

Methods: CCK- 8 , the wound healing analysis, colony formation assay, immunofluorescence analysis, Western blotting, flow cytometry, and animal models were used in the current study. Results: The data demonstrated that melatonin could inhibit GC growth, proliferation, and invasion both in vivo and in vitro. Apoptosis and autophagy induced in a concentrationdependent manner is response to melatonin-induced ER stress. Melatonin induced the expression of apoptotic and autophagy-related proteins, which was markedly attenuated by the ER stress inhibitor 4-PBA and autophagy inhibitor 3-MA. In addition, we used the specific IRE1 inhibitor STF 083010, finding that inhibiting IRE1 could considerably relieve ER stress-induced autophagy activity, as revealed by the reduction of LC3-II and Beclin-1.

Conclusion: This study confirmed that melatonin-induced inhibition of GC cell proliferation is mediated by the activation of the IRE/JNK/Beclin1 signaling.

Keywords: melatonin, autophagy, endoplasmic reticulum stress, apoptosis, inositolrequiring $1 \alpha$, Jun $\mathrm{N}$-terminal kinase

\section{Introduction}

Gastric cancer (GC), as the fifth prevalent malignancy, is the third leading cause of cancerrelated mortality worldwide. ${ }^{1}$ In spite of more attention shifted to the screening and treatment of GC over the past few decades, it remains a devastating disease with an unsatisfactory survival rate. ${ }^{2}$ Even with the advance in the earlier analysis approaches and innovative therapeutic methods, there are a less than 5-year survival rate and a poor postsurgery quality of life in most patients. ${ }^{3}$ Hence, there is a serious requirement for comprehending the biological mechanism of novel and promising agents for the treatment of GC. 
The endoplasmic reticulum(ER) is a tubule dynamic network included in folding, the synthesis, and processing more than one-third of the entire cellular proteome. ER Homeostasis can be altered by several pathophysiological circumstances, such as acidosis, hypoxia, and nutrient deprivation can alter a resultant imbalance between ER capacity and protein-folding load and accumulating unfolded proteins in the ER, a state termed ER stress. ${ }^{4}$ Upon the initiation of ER stress, the activation of the unfolded protein response (UPR) results in managing ER stress and restoring homeostasis of ER by cells. The UPR is settled by 3 main sensors placed in the ER, known as Inositol-requiring transmembrane kinase/endonuclease $1 \alpha$ (IRE1 $\alpha$ ), protein kinase RNA-like ER kinase (PERK) and activating transcription factor 6 (ATF6), which are also involved in inducing autophagy upon ER stress. ${ }^{5}$ The UPR is an adaptive response to reestablishment cellular homeostasis. When UPR cannot sufficiently be copied with ER stress, the cell will undergo apoptosis or autophagy. ${ }^{6}$ Autophagy, is a homeostatic mechanism and a metabolic process, -that involves the sequestration and delivery of cellular proteins, organelles, and cytoplasmic components to the lysosome- which are ultimately recycled and degraded to meet the metabolic demands of the cells. ${ }^{7}$ Autophagy plays a vital role in the development and progressing of numerous cancers, including pancreatic cancer, ${ }^{8}$ lung cancer, ${ }^{9}$ and gastric carcinoma. ${ }^{10}$

Melatonin (N-acetyl-5-methoxytryptamine) is an indoleamine that is synthesized by the pineal gland, retina, brain, heart, and the gastrointestinal system. ${ }^{11}$ Reportedly, the main source of melatonin is the gastrointestinal tract, with estimated production over 400 times higher than that in the pineal gland. ${ }^{12}$ The indoleamine has great pharmacological promise as an antioxidant enzyme that can scavenge free radicals, and protect from oxidative damage. ${ }^{13,14}$ Previous studies has shown that melatonin can inhibit the proliferation of various human cancers, including leukemia, ${ }^{15,16}$ hepatocarcinoma, ${ }^{17}$ breast cancer, ${ }^{18}$ colorectal cancer, ${ }^{19,20}$ lung cancer, ${ }^{21,22}$ and gastric carcinoma. ${ }^{23,24}$ However, studies investigating the molecular link between the UPR and autophagy activation by melatonin in GC are sparse. Unraveling this relationship is critical for novel targets for $\mathrm{GC}$ treatment.

\section{Materials and Methods}

\section{Cell Lines and Treatment}

The human gastric cancer cell lines AGS and SGC-7901 were prepared from the Cell Bank of the Chinese Academy of Sciences (Shanghai, China). Cultivation of the cells was regularly performed in RPMI-1640 media with the supplementation of $10 \%$ fetal bovine serum (Gibco, USA). Moreover, streptomycin $(100 \mathrm{U} / \mathrm{mL})$ and penicillin $(100 \mathrm{U} / \mathrm{mL})$ were inserted in the abovementioned culture system which was set at $37^{\circ} \mathrm{C}$ in a moistened air under $5 \% \mathrm{CO} 2$.

Melatonin was bought from (St. Louis, MO, Sigma Aldrich, USA), dissolved at a concentration of $1 \mathrm{M}$ as a stock solution in DMSO, and diluted with culture medium to suitable concentrations prior to use. Antibodies against $\beta$-actin, LC3A/B, Beclin-1, Ki-67, JNK, and phosphor-JNK were obtained from Cell Signaling Technology (Danvers, MA, USA). The P62 antibody was from Proteintech (Rosemont, IL, USA).Antibodies consist of IRE1 $\alpha$, GRP78, Caspase-3, Bax, and Bcl-2 were obtained from Abcam (Cambridge, Massachusetts, USA). The antibody was from 4-PBA was bought from Sigma Aldrich. 3-methyladenine(3-MA) and STF-083010 were purchased from Med Chem Express.

\section{CCK-8 Assay}

GC proliferation was determined through Cell Counting Kit-8 assay (Beyotime Institute of Biotechnology, Shanghai, China). At first, we seeded 4000 cells into 96-well plates to attach completely. The next day, we added given concentrations of melatonin to the culture media for 24,48 , and $72 \mathrm{~h}$. Then, $10 \%$ Cell Counting Kit (CCK)-8 reagent was assigned to each well and the incubation was performed for the plates for $2-4 \mathrm{~h}$ at $37^{\circ} \mathrm{C}$. Subsequently, we took a spectrophotometer to measure the absorbance at $450 \mathrm{~nm}$ for each day.

\section{The Wound Healing Analysis}

We made a scratch with a sterile clear tip $(200 \mu 1)$ to simulate a wound. The scratch pictures were taken by an inverted fluorescence microscope (Nikon, Tokyo, Japan) for the time point of $0 \mathrm{~h}$. Afterwards, the cells were directed with different concentrations of melatonin in medium, separately. After 24 or $48 \mathrm{~h}$ of treatment, cell migration was seen via an optical microscope.

\section{Cell Migration and Invasion Assays}

The AGS cells' invasiveness was calculated utilizing 24well Boyden chambers covered by $10 \mu \mathrm{g}$ of Matrigel (BD Biosciences, Sparks, MD). The cells $\left(3 \times 10^{5}\right.$ cells) re-suspended in $200 \mu \mathrm{L}$ of FBS-free medium was inserted to each upper chamber with various concentrations of melatonin $(\mathrm{mM})$, and DMEM comprising $10 \%$ FBS was introduced to the lower section. For the 
migrating assessments, $6 \times 10^{4}$ cells were cultivated onto filters in a 24-well transwell chamber, and the cells were undergone to the similar drug concentration gradient similar to the invasion assay. After $48 \mathrm{~h}$, the cells were gently scraped off the transwell chamber's upper surface, and the cells on the lower surface were washed 3 times with PBS, then submerged in 4\% paraformaldehyde for 15 min. Then, crystal violet was utilized for staining cells for $15 \mathrm{~min}$. The cells then were rinsed 2 times with PBS. The stained cells were seen in a randomly chosen field of view and counted utilizing a $200 \mathrm{X}$ inverted microscope.

\section{Flow Cytometry}

Apoptosis was found utilizing the Annexin V-FITC kit. GC cells were cultivated in 6-well plates treating with diverse concentrations of melatonin for 2 days. Then, the cells were rinsed 2 times with cold PBS and re-suspended in 1x Binding Buffer. Afterward, $100 \mu \mathrm{l}$ of the solution $\left(1 \times 10^{5}\right.$ cells $)$ was transmitted to a tube and adding $5 \mu 1$ of Annexin V-FITC and $5 \mu 1$ of PI, the solution was incubated for $10-20 \mathrm{~min}$ in the darkness. Subsequently, the tubes were gently vortexed and were immediately examined with a flow cytometer (FACScan, BD Biosciences, USA).

\section{Western Blot Assay}

Following treatment with melatonin, all proteins within cells and tissues were harvested and lysed on ice in RIPA lysis buffer (Beyotime Institute of Biotechnology, Shanghai, China) comprising phenylmethylsulfonyl fluoride (Beyotime Institute of Biotechnology, Shanghai, China) and phosphatase inhibitor (Sigma-Aldrich) based on the instructions of the manufacturer. The protein content was calculated utilizing the BCA Protein Assay Kit (Beyotime, Shanghai, China). The equivalent protein for each specimen was detached by $8-12 \%$ SDSpolyacrylamide gel electrophoresis and transmitted to a PVDF membrane. Using corresponding primary antibodies, the membrane was blocked and incubated at $4^{\circ} \mathrm{C}$ overnight. Moreover, Horseradish peroxidase-conjugated secondary antibodies (1:5000) were employed for $1 \mathrm{~h}$ at room temperature. Enhanced chemiluminescence(ECL) detection kit (Thermo Fisher Scientifc, Inc.) was utilized for detecting the proteins, and antibody band densities were visualized via Image Lab software.

\section{Immunofluorescence Analysis}

AGS cells were fixed on sterile sheet glass and disposed of with melatonin for $48 \mathrm{~h}$. Afterward, the cells were immersed in $4 \%(\mathrm{w} / \mathrm{v})$ paraformaldehyde for $20 \mathrm{~min}$ in PBS after permeabilization with $0.5 \%$ Triton X-100 for 10 min. Then the cells were blocked in $10 \%$ BSA for $1 \mathrm{~h}$, and incubated with primary antibody GRP78 and LC3-II overnight. The next day, the secondary antibody (1:200; cat\#: BYE026;Boyun, Shanghai, China)was added to the glass slides for $1 \mathrm{~h}$ at $37^{\circ} \mathrm{C}$. Finally, the cells were counterstained with DAPI (Beyotime, Shanghai, China) and visualized using a Leica DM4000B microscope (Jena, Germany).

\section{Immunohistochemistry}

Briefly, the tissue specimens were made constant in $4 \%$ paraformaldehyde solution, embedded in paraffin and then cut into a thickness of $4 \mu \mathrm{m}$. Sections were incubated with Ki67, Bax, and P62 antibodies at a respectively working dilution overnight. Subsequently, the slides were incubated with secondary antibody for $1 \mathrm{~h}$, rinsed with PBS and stained with 3, 3-diaminobenzidine tetrahydrochloride. Ultimately, the images were photographed by a light microscope (Leica, DM4000B).

\section{in vivo Tumorigenesis}

Male BALB/c nude mice at the age of 4 weeks were purchased from SLAC Co., Ltd. (Shanghai, China). The mice were kept under pathogen-free conditions with laminar airflow and controlled humidity and exposed to a $12 \mathrm{~h} \mathrm{light/dark}$ schedule. Before starting the experiment, the mice were acclimatized to the new environment for 1 week. Furthermore, the injection of SGC-7901 cells $\left(5 \times 10^{6}\right)$, suspended in $100 \mu \mathrm{L}$ sterile PBS, was performed subcutaneously into the right flank of the nude mice. The mice were monitored until 10 days for palpable tumor growth, the treatment group was treated with $50 \mathrm{mg} / \mathrm{kg}$ melatonin (ever day, intraperitoneal injection) containing $2.5 \%$ ethanol, while the control group received sterile saline. After treatment for 14 days, the mice were euthanized, and tissues were taken and were eliminated surgically and fixed in 4\% PFA/PBS solution. The animal protocols were performed in strict obedience of the instructions in the Guideline for IACUC of NIH and were approved by the Ethical Committee of Wenzhou Medical University and the Laboratory Animal Management Committee of Zhejiang Province (Approval ID: wydw2019-0433).

\section{Statistical Analysis}

All statistical analyses were performed utilizing the Prism5.0 software and the SPSS 15.0 statistical software. The statistical variations within two independent groups 
were determined via Student's $t$-test tests. To compare the means between groups, one-way analysis of variance (ANOVA) was conducted. The results were stated as the mean \pm standard deviation (SD) of at least 3 independent. The p-value of less than 0.05 was regarded a significant.

\section{Results}

\section{Melatonin Inhibited the Growth and} Migration Ability of Gastric Cancer Cells in vitro

To investigate the impact of melatonin on human GC cell lines, AGS and SGC-7901, CCK-8 assays were performed (Figure 1A). As shown, melatonin reduced GC' cell proliferation in a dose-and-time-dependent manner. The half maximal inhibitory concentration (IC50) values were $3.5 \mathrm{mM}$ in AGS and $3.27 \mathrm{mM}$ in SGC-7901. As for cells migration ability, we treated AGS cells with appropriate concentrations of melatonin for 48 and $72 \mathrm{~h}$. The scratch assay demonstrated that melatonin strongly inhibits cell migration (Figure 1B). Consistent with the scratch assay results, Transwell migration assays and Matrigel invasion assays (Figure 2A and B) unveil inhibiting the migration and invasion of AGS cells by melatonin. Quantitative analysis of the result is shown in Figure 2C. The melatonin could distinctly inhibit the AGS cells' migration and invasion.

\section{Melatonin Induced Apoptosis in Human GC Cell Line AGS}

To validate apoptotic cell death induced by melatonin, flow cytometry analysis was used. As observed in Figure 3A, the apoptosis rates after $48 \mathrm{~h}$ of treatment with $0,1,2,3 \mathrm{mM}$ melatonin were $6.45 \pm 0.055,13.49 \pm 0.930,24.48 \pm 0.167$ and
A

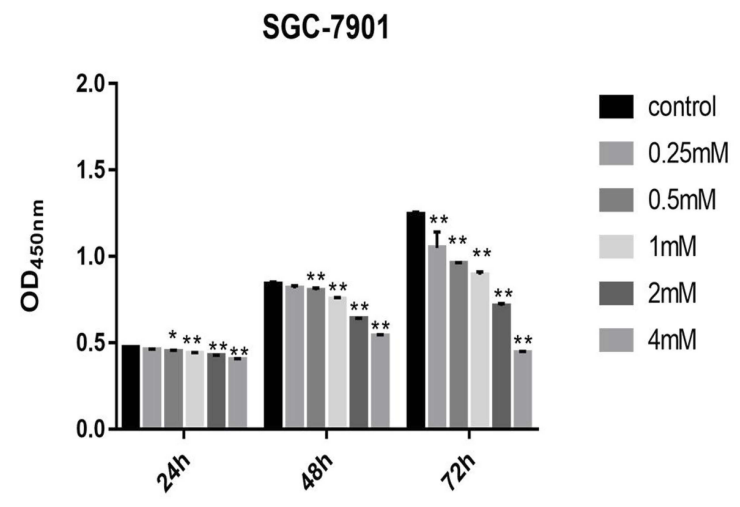

B

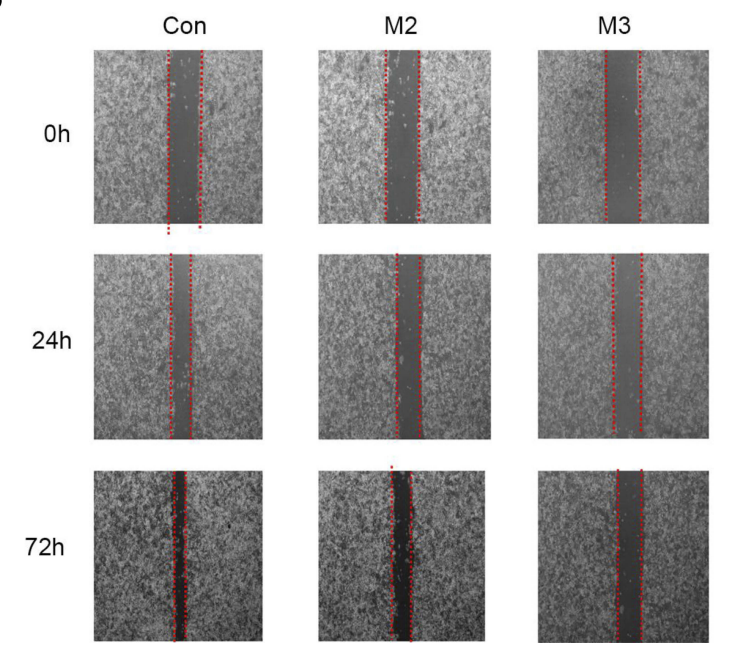

AGS
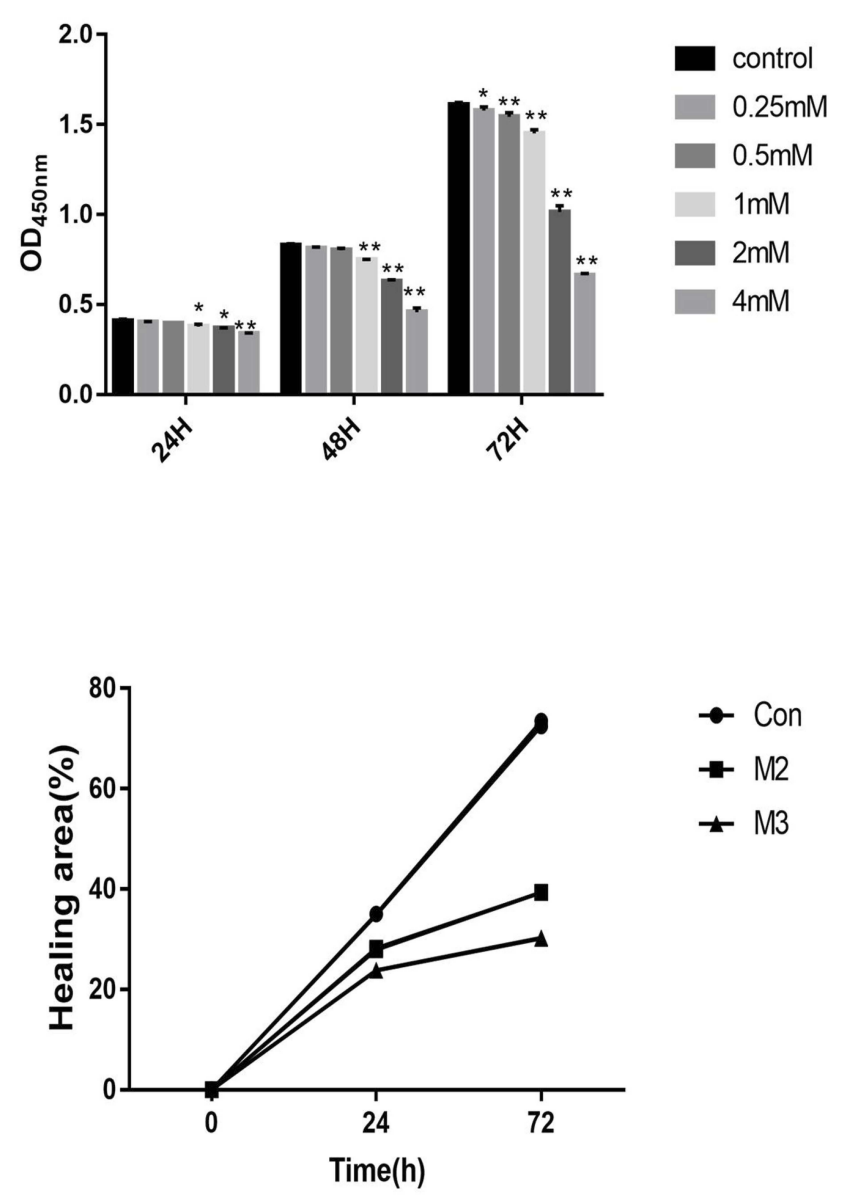

Figure I Melatonin inhibited the growth and migration ability of tumor cells (A) CCK8 assessment results of AGS and SGC-790 I cell activity following being treated with melatonin for 24,48 , and $72 \mathrm{~h}$ at varying concentrations. (B) Scratch assessment results indicated that the cell migration inhibition in the melatonin group. The figure indicates data as the means $\pm S D, n=3$. Student's $t$-test was compared to the control, $* P<0.05, * * P<0.01$.

Abbreviations: MI, I mM melatonin; M2, 2 mM melatonin; M3, 3 mM melatonin; Con, control trip. 
A

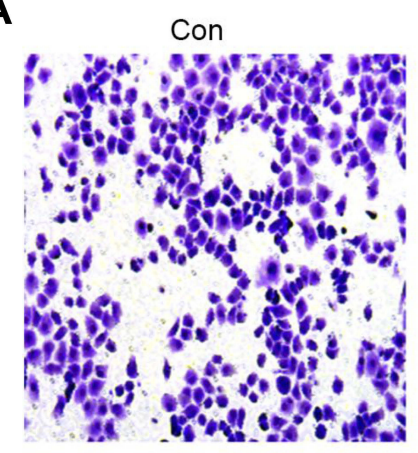

M1

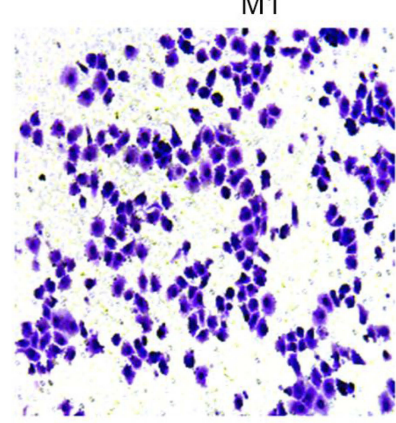

M2

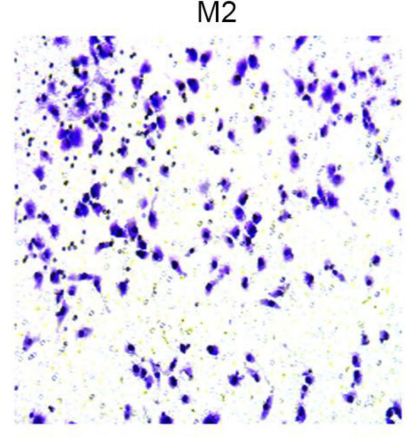

M3

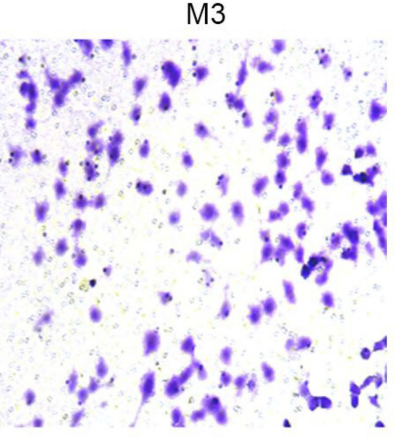

B
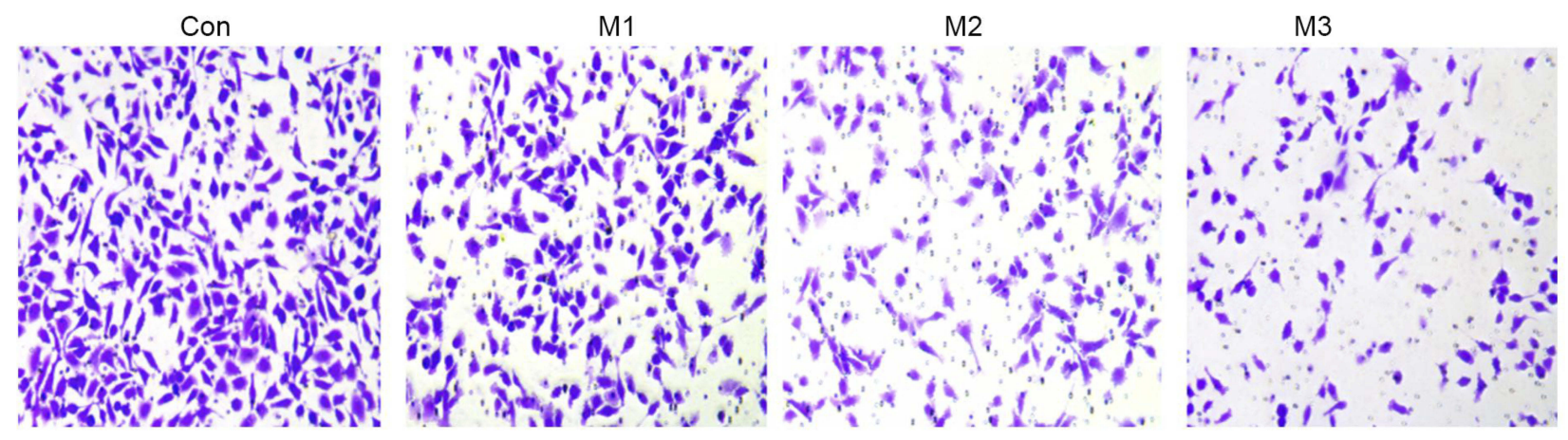

C

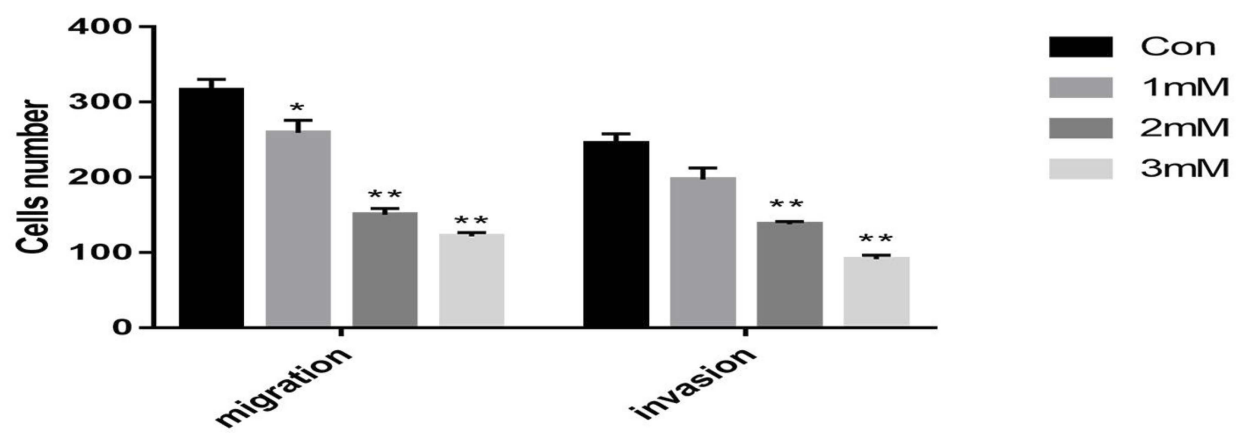

Figure 2 Melatonin inhibited the migration and invasion ability of tumor cells (A, B) Following treatment of AGS cells with increasing doses of melatonin (0, I, 2, 3 mM) for $48 \mathrm{~h}((\mathbf{A})$ for migration and (B) for invasion), migration and invasion assays were observed (magnification, $\mathbf{2 0 0}$ ). (C) Quantitative analysis of migration and invasion assays. Data as the means $\pm S D, n=3$. $* P<0.05, * * P<0.01$ compared to the control.

Abbreviations: MI, I mM melatonin; M2, 2 mM melatonin; M3, 3 mM melatonin; Con, control trip.

$30.5 \pm 0.458 \%$, respectively. Furthermore, the apoptosis impact of melatonin was explored by Western Blot analysis. The protein levels of apoptosis markers Bax and Caspase 3 were upregulated with increasing concentrations of melatonin, however the anti-apoptotic protein $\mathrm{Bcl} 2$ expression was reduced (Figure 3B). Quantitative analysis of the Western Blot analysis is shown in Figure 3C. The above- mentioned results reveal that the activation of apoptotic signaling pathways in AGS cells can be modulated by melatonin.
Contribution of the ER Stress Response to the AGS Autophagy and Apoptosis Induced by Melatonin

GRP78/bip is a major ER molecular chaperone, which separated from the three ER stress transducers, leading to an increased protein expression when ERS occurring. ${ }^{25}$ The GRP78 levels were significantly increased in AGS cells in a dose-dependent manner(Figure 4A).To elucidate the impact of melatonin on autophagy flux in gastric cancer, the related 


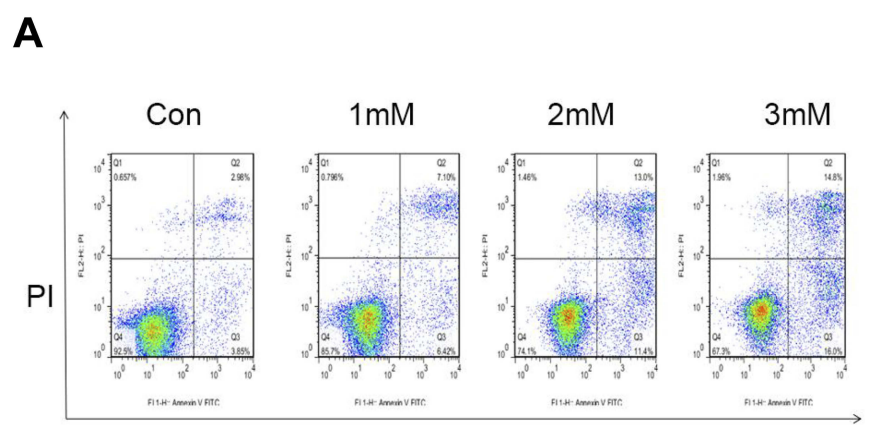

Annexin V-FITC

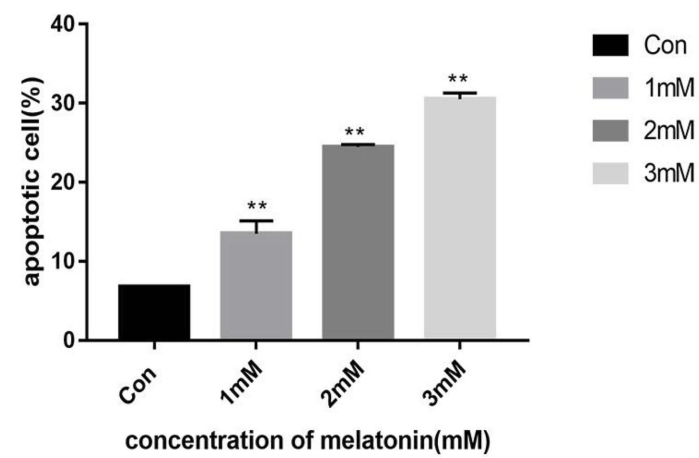

C
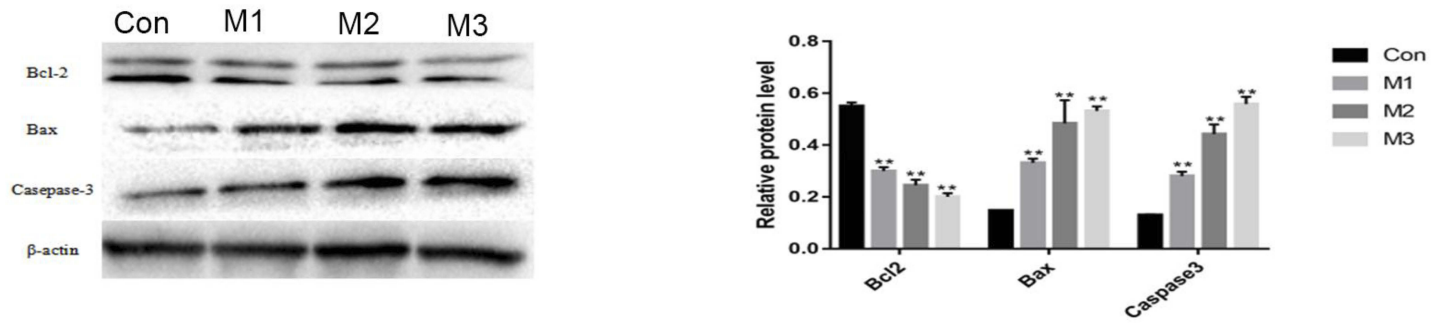

Figure 3 Melatonin- induced cell death of the human gastric cancer cell line AGS. (A) AGS cell apoptosis was specified using flow cytometry following treating with melatonin (0, I, 2, $3 \mathrm{mM})$ for $48 \mathrm{~h}$. (B) Western blot results of apoptosis-related proteins Bax and Casepase-3. $\boldsymbol{\beta}$-actin and Bcl-2 were utilized as the internal control. (C) Western blot results were measured and provided as the control percent. Data are expressed as mean \pm SD. **p $<0.01$ compared to control.

Abbreviations: MI, I mM melatonin; M2, 2 mM melatonin; M3, $3 \mathrm{mM}$ melatonin; Con, control trip.

hallmarks was tested through Western Blotting. Based on Figure 4A, melatonin-treated AGS cells expressed less p62 but more Beclin-1 compared to the equivalent control group. Additionally, LC3A/B-II expression in the AGS cell line was influenced by melatonin treatment. Furthermore, consistent with these findings, the protein expression of LC3 was further approved by the immunofluorescence results (Figure 5). In total, these findings revealed that melatonin activated autophagy in gastric cancer cells. To more demonstrate the promotion of apoptosis and autophagy by melatonin through ER stress, the specific ER stress inhibitor 4-PBA $(1 \mathrm{mM})$ and autophagy inhibitor 3-MA were added to AGS cells after melatonin exposure for $24 \mathrm{~h}$. 4-PBA is a chemical chaperone contributing to protein trafficking and folding within the ER, acting as an ER stress inhibitor alleviating ER stress. ${ }^{26}$ Of note, after exposure to 4-PBA for $2 \mathrm{~h}$, the expressions of GRP78, Beclin-1, LC3-II, and Bax were notably suppressed by 4 PBA (Figure 4B). Furthermore, 3-MA pretreatment expressed a markedly decrease in protein expression of GRP78, Beclin-1, LC3-II, Bax, and increase in Bcl-2 protein compared with melatonin-treated cells without 3-MA pretreatment. Quantitative analysis of the Western Blot analysis is shown in Figure 4D-F. These findings indicated that apoptosis is induced by melatonin through activating the autophagy and ER stress and autophagy. Nevertheless, the melatonin autophagy impact may be dominated by activating the ER stress.

\section{Promoting AGS Cells Apoptosis and Autophagy by Melatonin Through Activating IRE-JNK-Beclin I Pathway Activation}

Once ER stress is set off, JNK phosphorylates the antiapoptotic protein $\mathrm{Bcl}-2$; this phosphorylation results in the destruction of the Bcl-2/Beclin-1 complex and allows Beclin-1 to trigger autophagy. ${ }^{27}$ To investigate the IRE1 pathway's roles in the relationship between autophagy and the ER stress response, we used the specific IRE1 inhibitor STF-083010. The Western blot analysis showed that, pretreatment with STF-083010, inhibiting IRE1 could considerably relieve ER stress-induced autophagy activity, as revealed by the reduction of LC3-II and Beclin-1 (Figure 4C). The data suggested that melatonin promotes ER stress-induced autophagy through the IRE1/ JNK/Beclin-1 signaling pathway. 
A

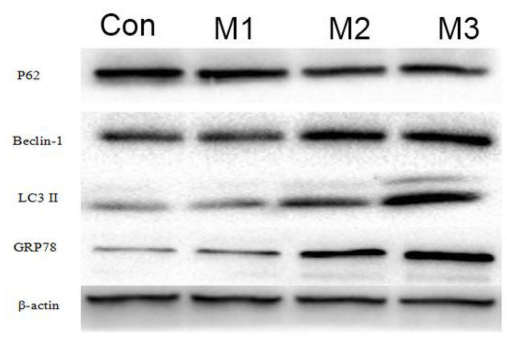

B

\section{Con M2 M2+3MA M2+4PBA}

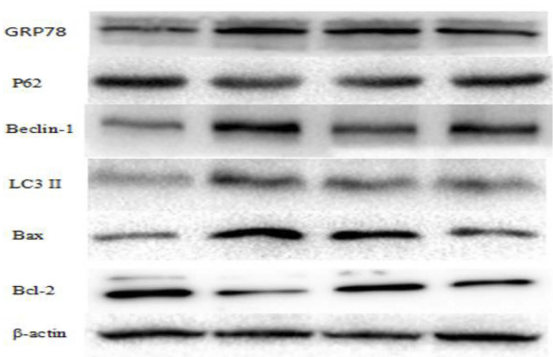

C

\section{Con M2 M2+STF}

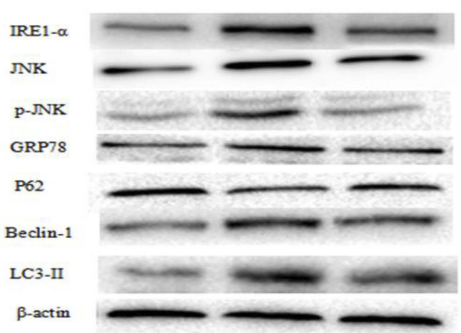

D

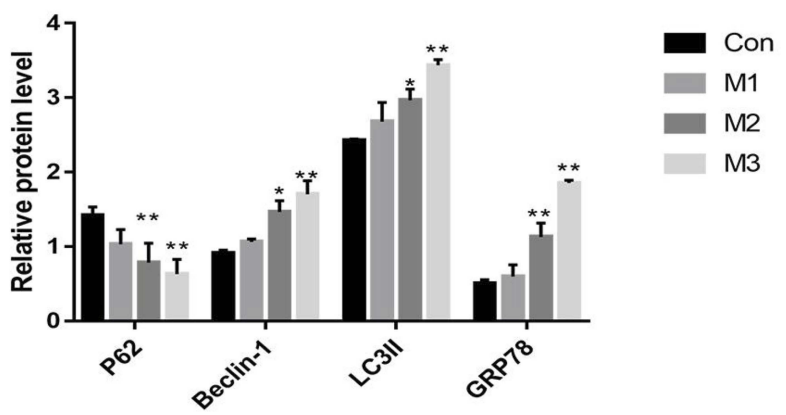

E

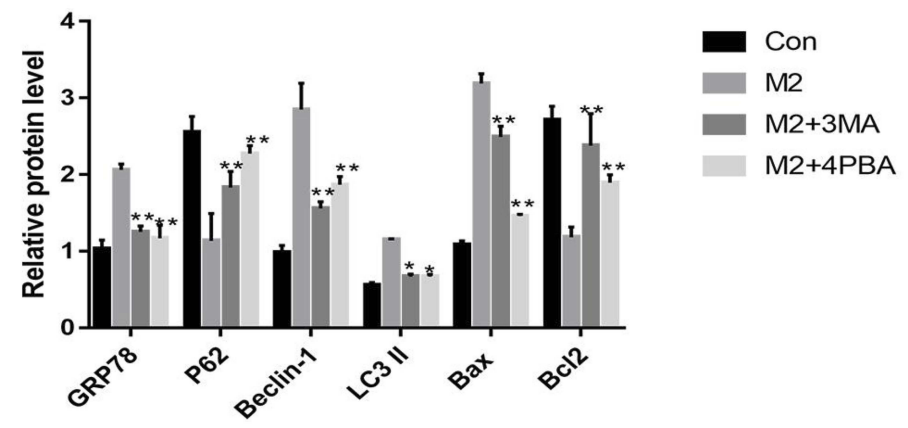

$\mathbf{F}$

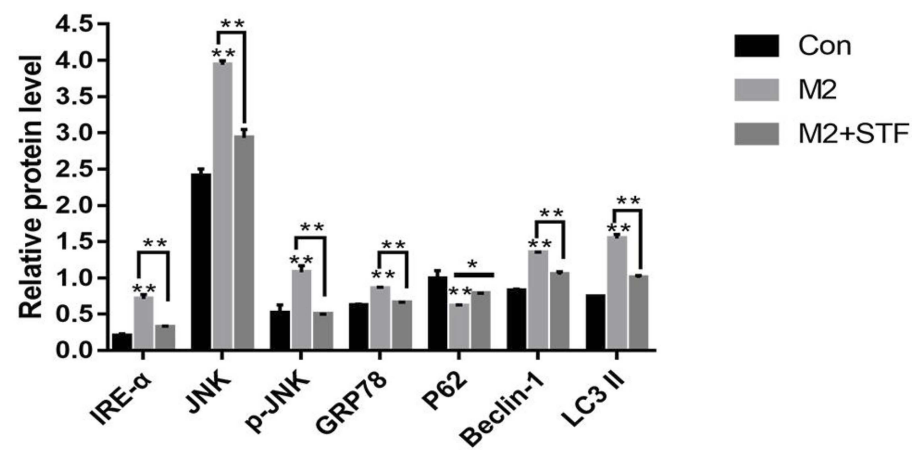

Figure 4 Melatonin increased speed of AGS cell apoptosis and autophagy by ER stress-upregulation. (A) Western blot analysis of the ER stress associated with protein expressions. Following the melatonin treatment, the GRP78 expressions significantly upregulated compared with the control group in a dose-dependent manner. $\boldsymbol{\beta}$-actin was utilized for normalization of band density as the loading control. (B) Western blot analysis of ER stress and autophagy related proteins expression following treating with the ER stress inhibitor 4- PBA and autophagy inhibitor 3-MA. (C) Western blot analysis of protein expression following treating with the IRE inhibitor STF 0830I0. It was found that GRP78, P62, Beclin-I, P-JNK, and LC3II expression was significantly upregulated in comparison with the control group, and, since $2 \mathrm{mM}$ melatonin is given, the protein expression was inverted. (D-F) Western blot results were measured and provided as the percent of the control. All tests were performed three times. Here, data are presented as the means $\pm S D, n=3$. Student's $t$-test was compared to control. $* P<0.05, * * P<0.01$ compared to the control.

Abbreviations: GRP78, glucose-regulated protein 78; 4-PBA, 4-phenylbutyric acid; 3-MA, 3-Methyladenine; STF, STF-0830I0; M2, 2 mM melatonin; JNK, c-Jun N-terminal kinase; P-, phosphorylated; Con, control trip.

\section{Inhibition of the Gastric Cancers' Growth by Melatonin in vivo}

In the present work, considering the potent in vitro antitumor impacts of melatonin in GC, the therapeutic efficacy of melatonin in SGC-7901 xenograft models was investigated. The mice were treated with an i.p. injection of melatonin daily for 10 days $(50 \mathrm{mg} / \mathrm{kg}$ body weight; $\mathrm{n}=6$ ) (Figure 6A). The results suggested that melatonin inhibited tumor growth in xenograft models compared with the control group. Mechanistically, we found that melatonin significantly upregulated ER stress related apoptosis and autophagy proteins including GRP78,Bax, Beclin-1 and LC II, and downregulated Bcl-2 and P62 in tumor tissues (Figure 6B). Moreover, melatonin did not 
A

\section{GRP78}
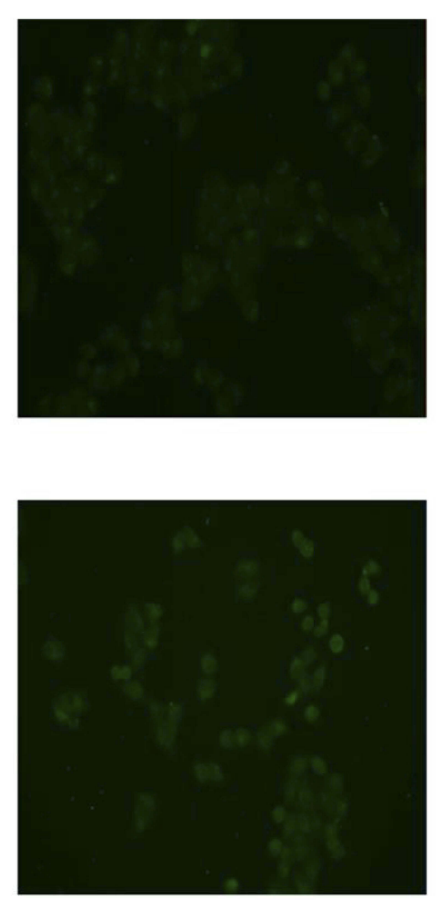

M2

B

\section{LC3}

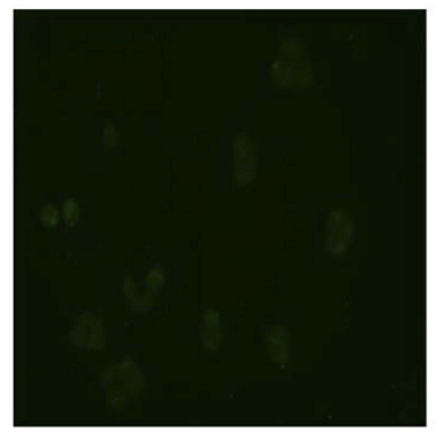

M2

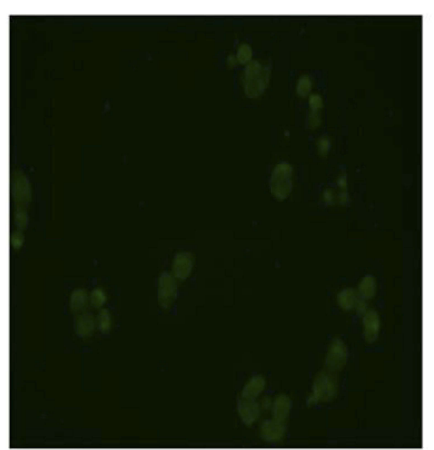

\section{DAPI}
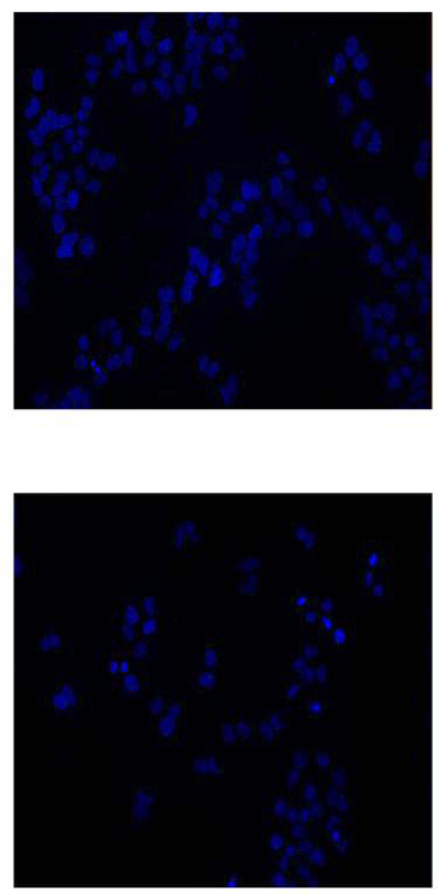

DAPI
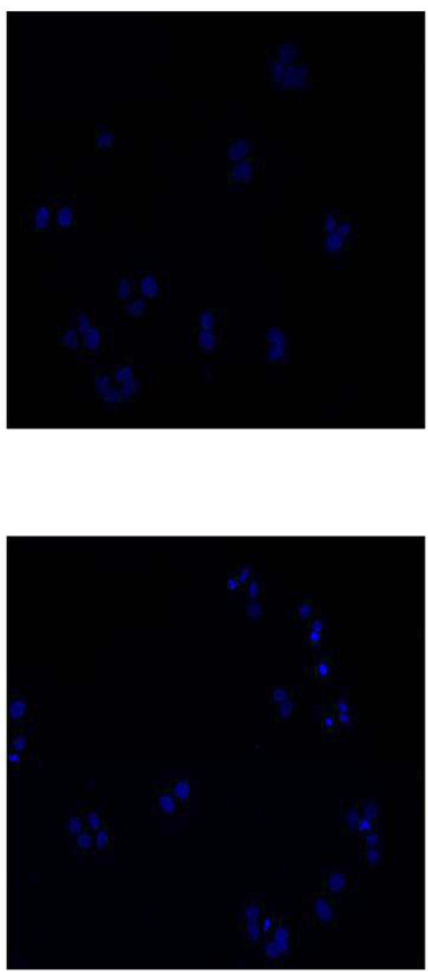

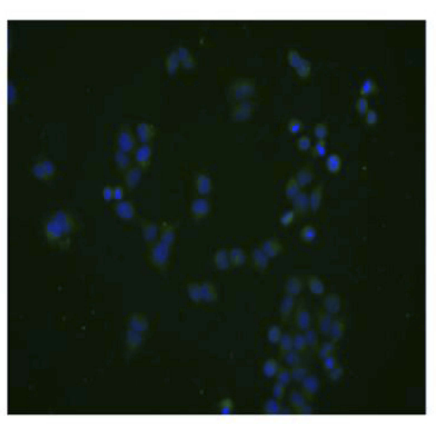

Merge

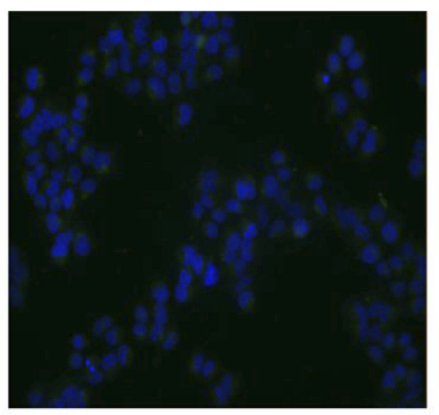

Merge
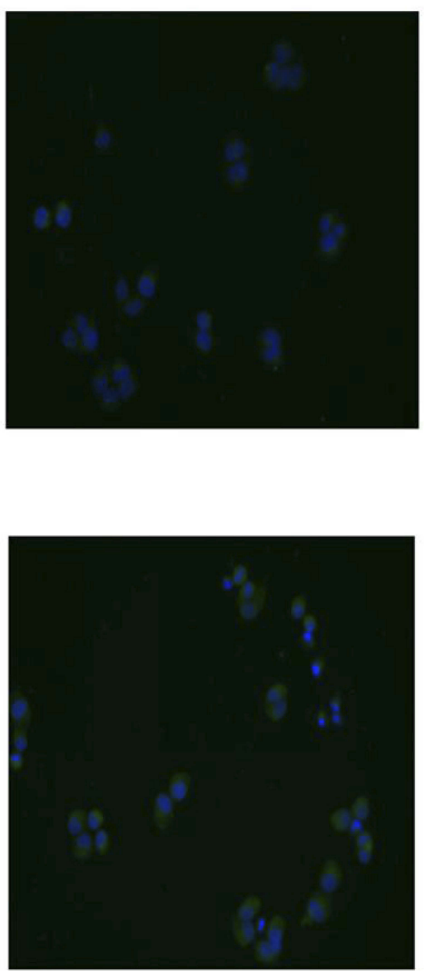

Figure 5 Melatonin activated autophagy by ER stress in GC cells (A, B) AGS cells were immunostained with GRP78 and LC3 antibodies and observed by the microscope (magnification, $\times 400$ ). Data are shown as means \pm SEM of at least 3 independent experiments. 
A
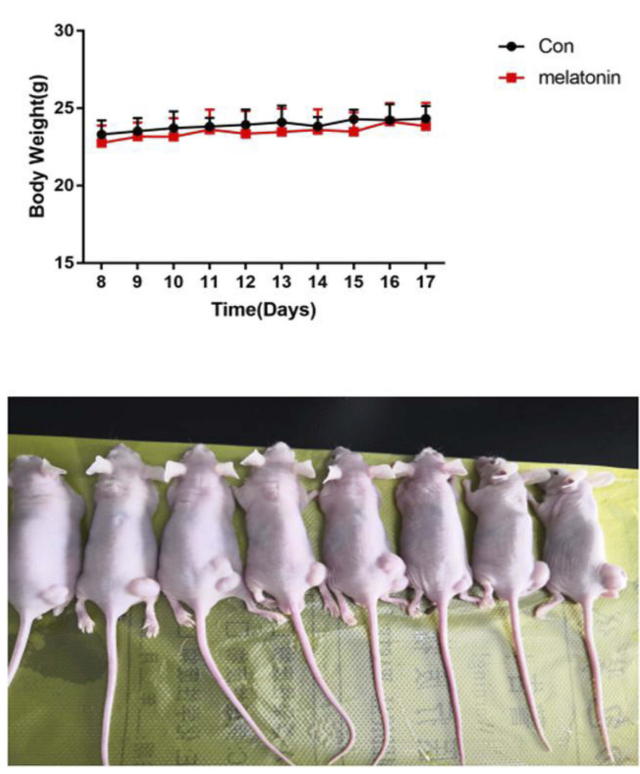

B

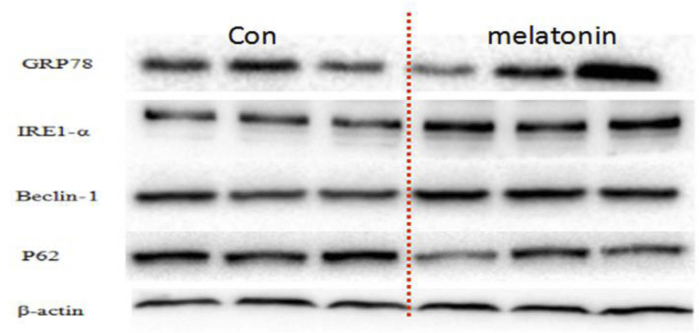

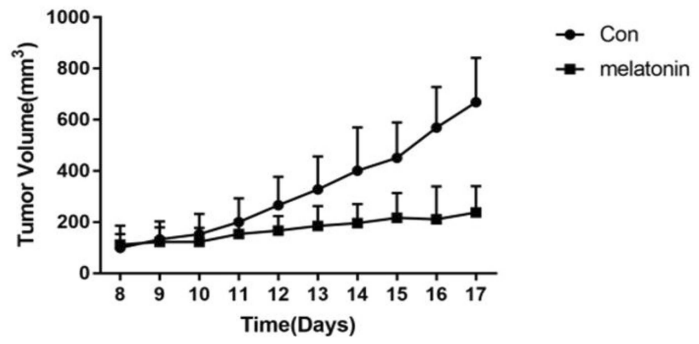

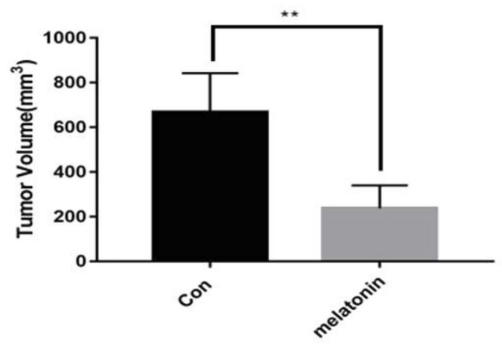

- Con

melatonin

D

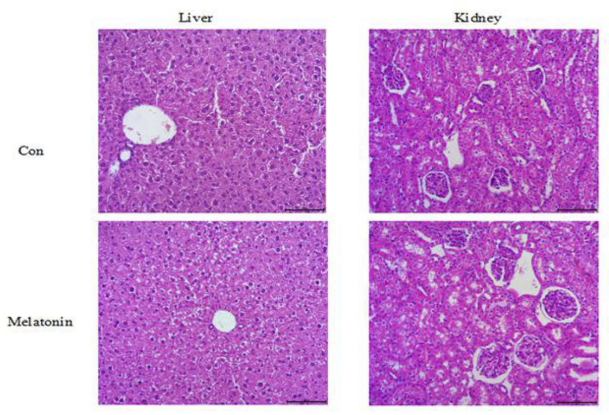

C

Ki-67

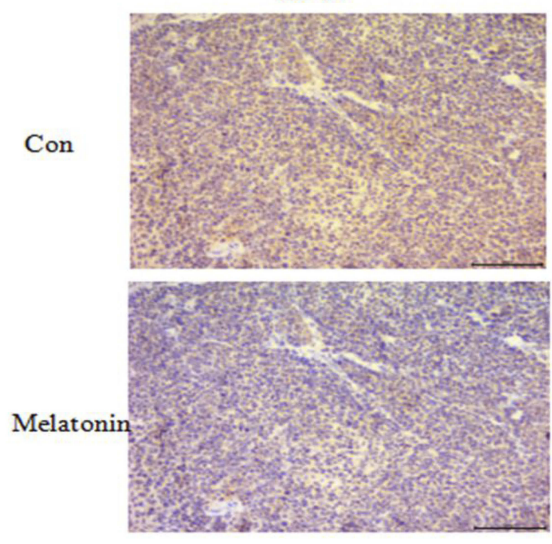

Bax

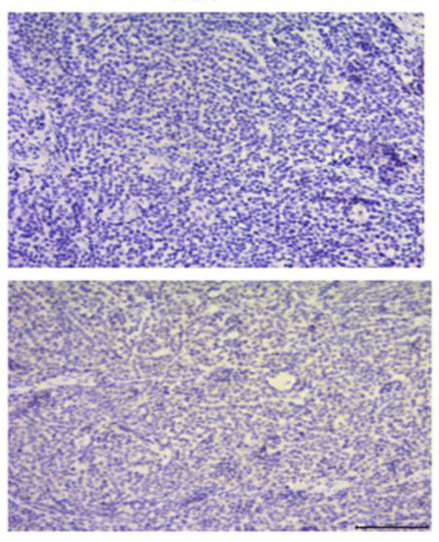

P62
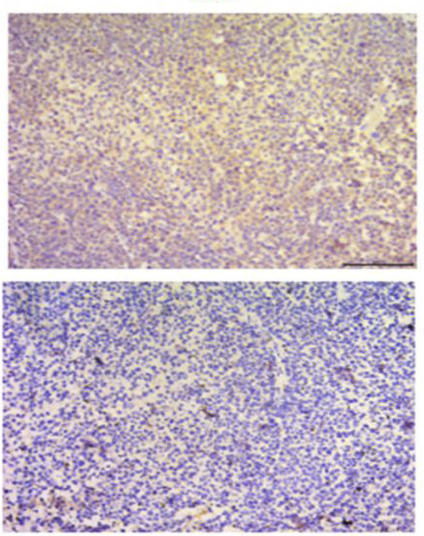

Figure 6 Melatonin prevents GC cells apoptosis, proliferation, and autophagy in vivo. (A) inoculation of 5x 106 SGC-790I cells was done into BALB/c-nude mice. The mice were assigned into two groups $(n=6)$ randomly, and they were treated with melatonin and PBS on a daily basis for 10 days. Measurement of body weight and tumor volumes was done daily. (B) The tumor tissues were cut out, and a Western blot assay was performed. (C) The apoptosis, autophagy and invasion of Bax, P62 and Ki67 in xenograft tumor tissues were determined by immunohistochemistry (magnification, $x 400$ ). (D) The kidneys and liver from the treatment and control groups were stained by eosin and hematoxylin so that the toxicity can be evaluated following the treatment. The kidney and liver's histological structures were obtained, and they were compared by the microscope (magnification, $\times 200)$. **P<0.0I compared to the control. Abbreviations: PBS, phosphate-buffered saline; Con, control. 
have considerable toxic side effects. No considerable difference was found in the weight of the mouse within the control and melatonin-treated group. Melatonin treatment caused no apparent toxicities on the liver and kidney and lung compared with the control group (Figure 6D). Additionally, using immunohistochemistry, the proteins expression levels associated with apoptosis, proliferation, and autophagy in xenograft tumor tissues were assessed. Figure 6C showed that apoptosis and autophagy were activated in xenograft tumors by melatonin. Ki67 and Bax revealed the existence of further apoptotic cells and clearly fewer proliferative cells in melatonin-treated tumors. In addition, the higher autophagy level, as calculated by P62, was detected in tumors in the melatonin treatment group.

\section{Discussion}

GC is the second most predominant kind of cancer occurrence and the second prominent cause of cancer-related mortality in China. ${ }^{28}$ Despite advancements in the therapeutic and surgical approaches, the five-year survival rate is only $5 \%-15 \%$. Previous studies show that melatonin, an indoleamine, plays a pivotal part in cancer-fighting. ${ }^{29}$ Numerous studies have shown that Melatonin has a significant inhibitory effect on the various human cancers at drug concentrations in different pathophysiological situations. ${ }^{30}$ The study aimed to investigate the possible signaling pathways involved in the antitumor impacts of the melatonin in human GC cells, which would lay a foundation for the development of new antitumor.

Emerging evidence has demonstrated that ER stress is related to cancer cell apoptosis and autophagy. ${ }^{31}$ The study of Ogata $\mathrm{M}$ et al indicated that there was a close relation between targeting the autophagy mechanisms and ER stress pathway. ${ }^{32}$ ER stress and autophagy seems to be critical for oncogenesis and cell homeostasis. Melatonin, a naturally occurring compound with clear potent inhibitory effects on cancer cells is one of the main candidates, which can be recruited herein. In preceding investigations on GC cells, melatonin was demonstrated as effective in arresting tumor cell growth. Hence, we assumed that autophagy and ER stress were involved in melatonininduced apoptosis in human GC cells. However, the specific signaling pathways included in the antitumor impacts of the melatonin remain unclear so far. More investigations are required better comprehending the interference among autophagy, ER stress, and apoptosis and to obviously outline the mechanisms responsible for modulating these responses by melatonin.

In this study, the percentage of apoptotic cells were dose-dependently incremented in GC cells treated with melatonin in comparison with the control group, as determined by the decreased cell activity responsible for inhibiting the cell migration. The findings essentially approved that melatonin prevented the cancer cell growth in vitro and indicated the potential anti-cancer impacts on gastric carcinoma. In the basic molecular mechanisms, ER stress was accelerated by melatonin as confirmed by the ER stress-related proteins' upregulated expressions. This was consistent with the melatonin's activated apoptosis impacts. To study the ER stress role caused by melatonin, in the present work the ER stress inhibitor 4-PBA was used to overwhelm the ER stress-related proteins. Particularly, melatonin treatment upregulated the ER stress-related proteins inhibited by 4-PBA. Consequently, there was a close relation between the melatonin enhanced AGS cell apoptosis and the ER stress activation.

Autophagy is vital for the maintenance of cellular homeostatic status since it sequesters damaged organelles and misfolded proteins and delivers them to lysosomes for degradation and recycling. ${ }^{33}$ Autophagy might be induced in cancer cells exposed to different perturbations, such as starvation, hypoxia, radiation, and growth factor withdrawal. ${ }^{34}$ It was found that treating the cancer cells with melatonin enhances the creation of autophagosome in AGS cells. During autophagy, LC3-I, a cytosolic form, is conjugated to LC3-II, which is employed to the autophagosome membrane. ${ }^{35}$ The accumulation of LC3-II is commonly used as markers of autophagy. Beclin-1 is required for the initiation of the autophagy process and is an interactive protein of PI3KC3, a specific target of 3-MA. ${ }^{25}$ The expressions of Beclin-1 and LC3 were considerably upregulated by melatonin, meanwhile the p62 expression was decreased. To discover the role of melatonin-induced autophagy in GC, we treated cells either with melatonin alone or joined with autophagy inhibitor 3-MA. Notably, these data indicated that the melatonin's anticancer action is enhanced by inducing the autophagy through incremented apoptosis of gastric cancer cells. In addition, melatonin could not reserve the autophagy activation in cells with 4-PBA-suppressed ERstress action. Hence, in this work, it concluded that melatonin enhanced apoptosis by activating the autophagy, while melatonin-induced autophagy triggered after the ER stress promotion.

GRP78 is a vital ER molecular chaperone and generally binds to IRE1 $\alpha$ in a regular physiological state. ${ }^{25}$ 
Under ER stress, IRE1 $\alpha$ is released, resulting in transautophosphorylation and oligomerization of IRE1 $\alpha$. The XBP1 mRNA is cleaved by the activated IRE1 $\alpha$ into its active spliced form (XBP1s), that are the transcription factors for different UPR genes contributing to apoptosis or autophagy. ${ }^{36}$ IRE1 $\alpha$ could recruit tumor necrosis factor receptor associated factor 2 (TRAF2), activate apoptosis signal-regulating kinase 1 (ASK1), and then phosphorylate c-Jun amino terminal kinase (JNK) at the ER. The release of Beclin-1 from Bcl-2 is stimulated by activated JNK, thus promoting vesicle nucleation and allowing autophagy to proceed. ${ }^{31}$ The present study investigates that ER stressinduced autophagy activity, as revealed by the levels of LC3-II and Beclin-1, were significantly reduced, following by the pretreatment with STF 083010.

\section{Conclusion}

In total, the present study revealed that the melatonin-induced promotion of $\mathrm{GC}$ cell proliferation was mediated via activation of the IRE/JNK/Beclin1 signaling pathway. Considering the role of melatonin on GC progression, the indoleamine may be a powerful candidate for antitumor treatments.

\section{Acknowledgments}

This study was based on the project approved by the Natural Science Foundation of Zhejiang Province (No. LQ17H030004) and Basic Public Welfare Research Project of Zhejiang Province (LGF18H160036).

\section{Disclosure}

The authors report no conflicts of interest in this work.

\section{References}

1. Bray F, Ferlay J, Soerjomataram I, Siegel RL, Torre LA, Jemal A. Global cancer statistics 2018: GLOBOCAN estimates of incidence and mortality worldwide for 36 cancers in 185 countries. CA Cancer J Clin. 2018;68(6):394-424. doi:10.3322/caac.21492

2. Camargo MC, Kim W-H, Chiaravalli AM, et al. Improved survival of gastric cancer with tumour Epstein-Barr virus positivity: an international pooled analysis. Gut. 2014;63(2):236-243. doi:10.1136/gutjnl-2013304531

3. Zou P, Xia Y, Chen T, et al. Selective killing of gastric cancer cells by a small molecule targeting ROS-mediated ER stress activation. Mol Carcinog. 2016;55(6):1073-1086. doi:10.1002/mc.v55.6

4. Walter P, Ron D. The unfolded protein response: from stress pathway to homeostatic regulation. Science (New York, NY). 2011;334 (6059):1081-1086. doi:10.1126/science.1209038

5. Okada T, Yoshida H, Akazawa R, Negishi M, Mori K. Distinct roles of activating transcription factor 6 (ATF6) and double-stranded RNA-activated protein kinase-like endoplasmic reticulum kinase (PERK) in transcription during the mammalian unfolded protein response. Biochem J. 2002;366(Pt 2):585-594. doi:10.1042/bj20020391
6. Peng X, Tu Y, Fu S, et al. 14-Deoxycoleon U-induced endoplasmic reticulum stress-mediated apoptosis, autophagy, and cell cycle arrest in lung adenocarcinoma. Onco Targets Ther. 2019;12:5955-5965. doi:10.2147/OTT.S211933

7. Pattingre S, Tassa A, Qu XP, et al. Bcl-2 antiapoptotic proteins inhibit Beclin 1-dependent autophagy. Cell. 2005;122(6):927-939. doi:10.1016/j.cell.2005.07.002

8. Liu W, Wang X, Sun J, Yang Y, Li W, Song J. Parthenolide suppresses pancreatic cell growth by autophagy-mediated apoptosis. Onco Targets Ther. 2017;10:453-461. doi:10.2147/OTT

9. Wang J, Li J, Cao N, Li Z, Han J, Li L. Resveratrol, an activator of SIRT1, induces protective autophagy in non-small-cell lung cancer via inhibiting Akt/mTOR and activating p38-MAPK. Onco Targets Ther. 2018;11:7777-7786. doi:10.2147/OTT

10. Yin S, Miao Z, Tan Y, et al. SPHK1-induced autophagy in peritoneal mesothelial cell enhances gastric cancer peritoneal dissemination. Cancer Med. 2019;8(4):1731-1743. doi:10.1002/cam4.2019.8.issue-4

11. Acuna-Castroviejo D, Escames G, Venegas C, et al. Extrapineal melatonin: sources, regulation, and potential functions. Cel Mol Life Sci. 2014;71(16):2997-3025.

12. Jaworek J, Leja-Szpak A, Nawrot-Porabka K, et al. Effects of melatonin and its analogues on pancreatic inflammation, enzyme secretion, and tumorigenesis. Int J Mol Sci. 2017;18:5. doi:10.3390/ ijms 18051014

13. Bonnefont-Rousselot D, Collin F, Jore D, Gardes-Albert M. Reaction mechanism of melatonin oxidation by reactive oxygen species in vitro. J Pineal Res. 2011;50(3):328-335. doi:10.1111/jpi.2011.50. issue-3

14. Poeggeler B, Saarela S, Reiter RJ, et al. Melatonin-a highly potent endogenous radical scavenger and electron donor: new aspects of the oxidation chemistry of this indole accessed in vitro. Ann N Y Acad Sci. 1994;738:419-420. doi:10.1111/j.1749-6632.1994.tb21831.x

15. Perdomo J, Cabrera J, Estevez F, Loro J, Reiter RJ, Quintana J. Melatonin induces apoptosis through a caspase-dependent but reactive oxygen species-independent mechanism in human leukemia Molt-3 cells. J Pineal Res. 2013;55(2):195-206. doi:10.1111/jpi.2013.55.issue-2

16. Bejarano I, Redondo PC, Espino J, et al. Melatonin induces mitochondrial-mediated apoptosis in human myeloid HL-60 cells. J Pineal Res. 2009;46(4):392-400. doi:10.1111/jpi.2009.46.issue-4

17. Martin-Renedo J, Mauriz JL, Jorquera F, Ruiz-Andres O, Gonzalez P, Gonzalez-Gallego J. Melatonin induces cell cycle arrest and apoptosis in hepatocarcinoma HepG2 cell line. J Pineal Res. 2008;45 (4):532-540. doi:10.1111/jpi.2008.45.issue-4

18. Nooshinfar E, Safaroghli-Azar A, Bashash D, Akbari ME. Melatonin, an inhibitory agent in breast cancer. Breast Cancer. 2017;24 (1):42-51. doi:10.1007/s12282-016-0690-7

19. Wang J, Guo W, Chen W, et al. Melatonin potentiates the antiproliferative and pro-apoptotic effects of ursolic acid in colon cancer cells by modulating multiple signaling pathways. J Pineal Res. 2013;54 (4):406-416. doi:10.1111/jpi.12035

20. Leon J, Casado J, Ruiz SMJ, et al. Melatonin reduces endothelin-1 expression and secretion in colon cancer cells through the inactivation of FoxO-1 and NF- KB. J Pineal Res. 2014;56(4):415-426. doi:10.1111/jpi.12131

21. Plaimee P, Weerapreeyakul N, Thumanu K, Tanthanuch W, Barusrux S. Melatonin induces apoptosis through biomolecular changes, in SK-LU-1 human lung adenocarcinoma cells. Cell Prolif. 2014;47(6):564-577. doi:10.1111/cpr.2014.47.issue-6

22. Chao CC, Chen PC, Chiou PC, et al. Melatonin suppresses lung cancer metastasis by inhibition of epithelial-mesenchymal transition through targeting to Twist. Clin Sci. 2019;133(5):709-722. doi:10.1042/CS20180945

23. Song J, Ma SJ, Luo JH, et al. Melatonin induces the apoptosis and inhibits the proliferation of human gastric cancer cells via blockade of the AKT/MDM2 pathway. Oncol Rep. 2018;39(4):1975-1983. doi:10.3892/or.2018.6282 
24. Wang X, Wang B, Xie J, Hou D, Zhang H, Huang H. Melatonin inhibits epithelialtomesenchymal transition in gastric cancer cells via attenuation of IL1beta/NFkappaB/MMP2/MMP9 signaling. Int J Mol Med. 2018;42(4):2221-2228. doi:10.3892/ijmm.2018.3788

25. Ron D, Walter P. Signal integration in the endoplasmic reticulum unfolded protein response. Nat Rev Mol Cell Biol. 2007;8 (7):519-529. doi:10.1038/nrm2199

26. Zhang W, Chen L, Shen Y, Xu J. Rifampicin-induced injury in L02 cells is alleviated by 4-PBA via inhibition of the PERK-ATF4-CHOP pathway. Toxicol in vitro. 2016;36:186-196. doi:10.1016/j.tiv.2016.07.017

27. Wei Y, Pattingre S, Sinha S, Bassik M, Levine B. JNK1-mediated phosphorylation of $\mathrm{Bcl}-2$ regulates starvation-induced autophagy. Mol Cell. 2008;30(6):678-688. doi:10.1016/j.molcel.2008.06.001

28. Chen W, Zheng R, Baade PD, et al. Cancer statistics in China, 2015. CA Cancer J Clin. 2016;66(2):115-132. doi:10.3322/caac.21338

29. Xin Z, Jiang S, Jiang P, et al. Melatonin as a treatment for gastrointestinal cancer: a review. J Pineal Res. 2015;58(4):375-387. doi:10.1111/jpi.2015.58.issue-4

30. Fernandez A, Ordonez R, Reiter RJ, Gonzalez-Gallego J, Mauriz JL. Melatonin and endoplasmic reticulum stress: relation to autophagy and apoptosis. J Pineal Res. 2015;59(3):292-307. doi:10.1111/jpi.12264
31. Ogata M, Hino S-I, Saito A, et al. Autophagy is activated for cell survival after endoplasmic reticulum stress. Mol Cell Biol. 2006;26 (24):9220-9231. doi:10.1128/MCB.01453-06

32. Chen PP, Ma XY, Lin Q, et al. Kangfuxin promotes apoptosis of gastric cancer cells through activating ERstress and autophagy. Mol Med Rep. 2017;16(6):9043-9050. doi:10.3892/mmr.2017.7716

33. Dunn WA Jr. Studies on the mechanisms of autophagy: formation of the autophagic vacuole. J Cell Biol. 1990;110(6):1923-1933. doi:10.1083/jcb.110.6.1923

34. Lee YK, Jun YW, Choi HE, et al. Development of LC3/ GABARAP sensors containing a LIR and a hydrophobic domain to monitor autophagy. EMBO J. 2017;36(8):1100-1116. doi:10.15252/embj.201696315

35. Furuya N, Yu J, Byfield M, Pattingre S, Levine B. The evolutionarily conserved domain of Beclin 1 is required for $\mathrm{Vps} 34$ binding, autophagy and tumor suppressor function. Autophagy. 2005;1(1):46-52. doi:10.4161/auto.1.1.1542

36. Sano R, Reed JC. ER stress-induced cell death mechanisms. Biochim Biophys Acta. 2013;1833(12):3460-3470. doi:10.1016/j. bbamcr.2013.06.028

\section{Publish your work in this journal}

OncoTargets and Therapy is an international, peer-reviewed, open access journal focusing on the pathological basis of all cancers, potential targets for therapy and treatment protocols employed to improve the management of cancer patients. The journal also focuses on the impact of management programs and new therapeutic agents and protocols on patient perspectives such as quality of life, adherence and satisfaction. The manuscript management system is completely online and includes a very quick and fair peer-review system, which is all easy to use. Visit http://www.dovepress.com/ testimonials.php to read real quotes from published authors. 